\title{
ON LINE INTEGRALS OF RATIONAL FUNCTIONS OF TWO COMPLEX VARIABLES
}

\author{
E. AZOFF, K. CLANCEY AND I. GOHBERG ${ }^{1}$
}

\begin{abstract}
Let $\gamma$ be a simple rectifiable arc in the complex plane and $r(z, w)$ a rational function of two complex variables. Set $r_{\gamma}(z)=\int_{\gamma} r(z, w) d w$. The natural domain of $r_{\gamma}$ has countably many components, and $r_{\gamma}$ may vanish identically on infinitely many of these. It is shown however that unless $\gamma$ spirals in to one of its endpoints, only finitely many zeros of $r_{\gamma}$ are isolated.
\end{abstract}

0. Introduction. Let $\gamma$ be a rectifiable curve in the complex $w$-plane $\mathbf{C}_{w}$. If

$$
r(z, w)=\frac{p(z, w)}{q(z, w)}
$$

is a rational function which is the quotient of the relatively prime polynomials $p(z, w), q(z, w)$ in the two complex variables $z, w$, then on the domain

$$
\Phi_{\gamma}(r)=\left\{z \in \mathbf{C}_{z} \mid q(z, w) \neq 0, w \in \gamma\right\}
$$

we may define the analytic function

$$
r_{\gamma}(z)=\int_{\gamma} r(z, w) d w .
$$

When $\gamma$ is a Jordan curve, the residue theorem shows that $r_{\gamma}$ is a continuous algebraic function on $\Phi_{\gamma}(r)$, and consequently, all but finitely many of the zeros of $r_{\gamma}$ lie in components on which it vanishes identically. This result was presented in [1], where it was used in studying certain finite rank perturbations of matrix multiplication operators. These perturbation results find applications in the theory of one dimensional singular integral operators along contours in the complex plane.

In trying to extend such perturbation results to the case of nonclosed curves, it became necessary to investigate the zero sets of $r_{\gamma}$ for general rectifiable curves. We do this in the present paper, obtaining the following as our main result. We use the term arc to mean a simple curve.

THEOREM 1. Suppose $\gamma$ is a rectifiable arc and $r=r(z, w)$ is a rational function of the two complex variables $z, w$. Let $r_{\gamma}$ be the analytic function defined by $(0.3)$ on the domain $\Phi_{\gamma}(r)$ given in (0.2). If $\gamma$ does not spiral in to either of its endpoints, then only finitely many of the zeros of $r_{\gamma}$ can be isolated.

Received by the editors June 18, 1981 and, in revised form, December 23, 1981.

1980 Mathematics Subject Classification. Primary 30C15, 45B05.

Key words and phrases. Zero set, line integral, rational function, spiral.

'The work of the authors was supported by grants from the National Science Foundation.

(1982 American Mathematical Society 0002-9939/82/0000-0046/ $\$ 2.50$ 
The new feature introduced by considering nonclosed curves is the appearance of logarithmic singularities for $r_{\gamma}$. By not allowing $\gamma$ to spiral in to its endpoints, we force these logarithms to have bounded imaginary parts, and the proof of Theorem 1 can be made to depend on a property of such logarithms. On the other hand, when $\gamma$ does spiral in to an endpoint, the logarithmic singularities of $r_{\gamma}$ can have unbounded imaginary parts, and the conclusion of Theorem 1 can fail. This dichotomy struck us as an interesting function theory result, and we decided to separate the story on the zeros of $r_{\gamma}$ from its applications presented in [2].

The structure of the remainder of the paper is as follows. In $\S 1$, the necessary preliminaries on logarithms are presented. The proof of Theorem 1 is presented in $\S 2$, and in $\S 3$, a special arc is constructed to demonstrate what can happen when $\gamma$ does spiral in to an endpoint. In the final section of the paper, we exploit the proof of Theorem 1 to get an explicit local representation for $r_{\gamma}$.

The authors wish to thank Domingo Herrero for a time-saving conversation concerning the example in §3; we would also like to acknowledge the expository suggestions of both the referee and editor.

1. Preliminaries. In this section, we discuss the statement of Theorem 1 and establish some preliminary material for its proof. We begin by pointing out that the domain $\Phi_{\gamma}(r)$ can have infinitely many components, and the theorem makes no assertion concerning the nonisolated zeros of $r_{\gamma}$. Indeed, the following example exhibits a smooth arc $\gamma$ for which $r_{\gamma}$ vanishes identically on infinitely many of these components.

EXAmple 1. Let $\gamma:[-1,1] \rightarrow \mathbf{C}$ by

$$
\gamma(t)= \begin{cases}t & \text { if }-1 \leqslant t \leqslant 0 \\ t+i t^{4} \sin \frac{\pi}{t} & \text { if } 0<t \leqslant 1\end{cases}
$$

and take $r(z, w)=1 /(w+z)+1 /(w-z)+\pi i$. The domain $\Phi_{\gamma}$ consists of all $z \in \mathbf{C}$ for which neither $z$ nor $-z$ lies on the trace of $\gamma$. Thus a typical component of $\Phi_{\gamma}$ is bounded by an arch of the form $\left.\gamma\right|_{[1 /(n+1), 1 / n]}$ and a line segment on the $x$-axis. Let $\delta:[-1,1] \rightarrow \mathbf{C}$ by

$$
\delta(t)= \begin{cases}-\gamma(t) & \text { if }-1 \leqslant t \leqslant 0 \\ \gamma(t) & \text { if } 0 \leqslant t \leqslant 1\end{cases}
$$

Then $\delta$ is a closed curve and an easy change of variables shows that

$$
r_{\gamma}(z)=2 \pi i+\int_{\delta}\left(\frac{1}{w+z}+\frac{1}{w-z}\right) d w .
$$

Now the latter integral is $\pm 2 \pi i$, depending on which quadrant contains the (bounded) component in which $z$ lies. In particular, the set of components of $\Phi_{\gamma}(r)$ on which $r_{\gamma}$ vanishes identically is neither finite nor cofinite.

As usual, a branch of the logarithm function defined on a domain $\Omega$ in the complex sphere $\hat{\mathbf{C}}_{\zeta}=\mathbf{C}_{\zeta} \cup\{\infty\}$ will mean an analytic function $L$ defined on $\Omega$, and satisfying $\exp L(\zeta)=\zeta(\zeta \in \Omega)$. If $\Gamma$ denotes an arc joining the points 0 and $\infty$ in 
$\hat{\mathbf{C}}_{\zeta}$ and $\zeta=1$ is not on $\Gamma$, then we will denote by $L_{\Gamma}$ the branch of the logarithm function on $\hat{\mathbf{C}}_{\zeta} \backslash \Gamma$ satisfying $L_{\Gamma}(1)=0$.

Given an arc $\gamma$ in $\mathbf{C}_{w}$ with initial point $a$ and terminal point $b$, we will use the notation $\Gamma$ for the image of $\gamma$ in $\hat{\mathbf{C}}_{\zeta}$ under the Möbius transformation $\zeta(w)=$ $(b-w)(a-w)^{-1}$, and define $l_{\gamma}=L_{\Gamma} \circ \zeta$. We will say that $\gamma$ spirals in to an endpoint if the imaginary part of $L_{\Gamma}$ is unbounded. The terminology is appropriate in the sense that arcs which conform to one's intuitive notion of (direct) spiraling towards an endpoint do satisfy the condition; on the other hand, the formal definition also includes arcs which do too much 'meandering' to fit the stereotypic picture. In any case, Theorem 1 applies to a large class of arcs, including all smooth ones. (We follow the usual convention of calling a curve smooth if it can be reparameterized by a $C^{1}$ function.)

When the arc $\gamma$ is rectifiable, we have

$$
\int_{\gamma} \frac{d w}{w-z}=L_{\Gamma}\left(\frac{b-z}{a-z}\right)=l_{\gamma}(z)
$$

The proof of Theorem 1 will be based on the following result. We follow the usual conventions concerning the domains of compositions and algebraic combinations of functions, so that as in Example 1, the function $h$ of the proposition may vanish identically on infinitely many components of its domain. Note for future reference that taking $g_{1}$ to be constant gives $h$ a meromorphic summand.

Proposition 1. Let $f_{1}, \ldots, f_{n}$ and $g_{1}, \ldots, g_{n}$ be meromorphic functions defined in a neighborhood of $z=0$. Suppose $L_{1}, \ldots, L_{n}$ are branches of the logarithm function with bounded imaginary parts. Then there is a neighborhood of zero in which the function

$$
h(z)=\sum_{j=1}^{n} f_{j}(z) L_{j}\left(g_{j}(z)\right)
$$

has only finitely many isolated zeros.

PROOF. Let $\left\{z_{k}\right\}_{k=1}^{\infty}$ be a sequence of zeros of $h$ converging to zero. It suffices to show that $h$ must vanish on a neighborhood of some $z_{k}$.

Write $g_{j}(z)=z^{m_{j}} h_{j}(z)$, where $m_{j}$ is an integer and $h_{j}$ is analytic at zero with $h_{j}(0) \neq 0$. Choose a half-line joining 0 to $\infty$ disjoint from $\left\{z_{k}\right\}_{k=1}^{\infty}$ and $\left\{h_{j}(0)\right\}_{j=1}^{n}$. Let $L_{0}$ be a branch of the logarithm function on the complement of this half-line. Then for each $j, L_{j}\left(g_{j}(z)\right)$ and $L_{0}\left(h_{j}(z)\right)+m_{j} L_{0}(z)$ have the same derivative. Since the logarithms involved have bounded imaginary parts, the differences between these functions can take on only a finite number of values. Thus we can find an (in general disconnected) open set $U$ containing a subsequence of the sequence $\left\{z_{k}\right\}_{k=1}^{\infty}$ such that

$$
h(z)=\sum_{j=1}^{n} f_{j}(z)\left[L_{0}\left(h_{j}(z)\right)+m_{j} L_{0}(z)+2 \pi i l_{j}\right], \quad z \in U,
$$

where the $\left\{l_{j}\right\}$ are integers. Clearly, we can rewrite $h$ in the form

$$
h(z)=g_{0}(z)+f_{0}(z) L_{0}(z), \quad z \in U,
$$

where $f_{0}$ and $g_{0}$ are meromorphic at zero. 
Suppose $f_{0}$ and $g_{0}$ did not both vanish identically. By multiplying $h$ by an appropriate power of $z$, we could make $f_{0}$ and $g_{0}$ analytic at 0 , with not both of $f_{0}(0)$, $g_{0}(0)$ equaling zero. But then the finiteness of $\lim _{n \rightarrow \infty} f_{0}\left(z_{n}\right) L_{0}\left(z_{n}\right)$ would force $f_{0}(0)=0$, and since $\lim _{z \rightarrow 0} z L_{0}(z)=0$, we would have $g_{0}(0)=0$ as well. This contradiction proves that $h$ vanishes in $U$ and completes the proof.

2. Proof of the main result. We proceed to the proof of Theorem 1 . Let $\left\{z_{j}\right\}_{j=1}^{\infty}$ be a sequence of zeros for $r_{\gamma}$. The $\left\{z_{j}\right\}_{j=1}^{\infty}$ have an accumulation point in the Riemann sphere $\hat{\mathbf{C}}_{z}$ which, by means of an elementary substitution, can be assumed to be $z=0$. We will complete the proof by showing that $r_{\gamma}$ vanishes in a neighborhood of some $z_{j}$.

Fix a half line $H$ joining 0 to $\infty$ which misses the $\left\{z_{j}\right\}_{j=1}^{\infty}$. In a deleted neighborhood of $z=0$, it is possible to choose a complete set $w_{1}(z), w_{2}(z), \ldots, w_{n}(z)$ of distinct root functions for the equation $q(z, w)=0$, which possess expansions of the form

$$
w_{j}(z)=\sum_{m=-k}^{\infty} w_{m}^{(j)}\left[z^{1 / \alpha_{j}}\right]^{m}, \quad j=1, \ldots, n ;
$$

here $z^{1 / \alpha_{j}}$ denotes an analytic branch of the $\alpha_{j}$ th root of $z$ defined off $H$.

Taking $\alpha=\prod_{j=1}^{n} \alpha_{j}$, we then have $q\left(z^{\alpha}, w\right)=c \prod_{j=1}^{n}\left(w-w_{j}\left(z^{\alpha}\right)\right)$ where $c$ is a nonzero constant. Note that the functions $\left\{w_{j}\left(z^{\alpha}\right)\right\}$ are meromorphic in a neighborhood of zero, and $r_{\gamma}(z)$ will have only finitely many isolated zeros if and only if $r_{\gamma}\left(z^{\alpha}\right)$ does. Thus, by substituting $z=u^{\alpha}$ in $r(z, w)$, we see there is no loss of generality in assuming the root functions $w_{1}, \ldots, w_{n}$ are themselves meromorphic in a neighborhood $\Delta$ of $z=0$. By performing a partial fraction expansion of (the new) $r(z, \cdot)$, we obtain

$$
r_{\gamma}(z)=g_{0}(z)+\int_{j=1}^{n} f_{j}(z) \int_{\gamma} \frac{d w}{w-w_{j}(z)}, \quad z \in \Delta \cap \Phi_{\gamma}(r),
$$

where $g_{0}, f_{1}, \ldots, f_{n}$ are meromorphic at $z=0$. (Some of the $f_{j}$ 's may vanish identically.)

Applying Equation (1.1) to (2.1), we conclude that $r_{\gamma}(z)$ has the form (1.2). Thus appealing to Proposition 1, we see that $r_{\gamma}$ vanishes in a neighborhood of some $z_{j}$, and the proof is complete.

REMARKS. If $\gamma_{1}, \ldots, \gamma_{m}$ and $r_{1}, \ldots, r_{m}$ satisfy the hypotheses of Theorem 1 , then $\sum_{j=1}^{m} r_{\gamma_{j}}$ has but finitely many isolated zeros; only minor variations in the above proof are required. In particular, if $\gamma$ is any smooth curve, then $\gamma$ can be decomposed as the finite union of simple arcs, and thus $r_{\gamma}$ satisfies the conclusion of the theorem; as the curve $\delta$ of Example 1 shows, such a $\gamma$ can have infinitely many self-intersections.

3. An example. In this section, we show that the conclusion of Theorem 1 can fail if $\gamma$ spirals in to an endpoint. This can be seen by taking $\gamma$ as in the following proposition and $r(z, w)=1 /\left(w-z^{2}\right)+1 / z$.

PROPOSITION 2. There is a rectifiable arc $\gamma$ such that the equation $\int_{\gamma} d w /\left(w-z^{2}\right)=$ $1 / z$ has infinitely many isolated solutions. 
Proof. Our arc $\gamma$ will have initial point $w=1$ and terminal point $w=0$. The proposition is equivalent to a statement concerning logarithms. Indeed, if $\Gamma$ is the image of $\gamma$ under the Möbius transformation $\zeta(w)=w /(w-1)$, then from (1.1) we have $\int_{\gamma} d w /\left(w-z^{2}\right)=L_{\Gamma}\left(z^{2} /\left(z^{2}-1\right)\right)$. Thus the proposition can be established by constructing a branch cut $\Gamma$ for which $\gamma=\zeta^{-1}(\Gamma)$ is rectifiable and the equation $z L_{\Gamma}\left(z^{2} /\left(z^{2}-1\right)\right)=1$ has infinitely many solutions.

Let $k$ be a positive integer, and write $L_{0}$ for the principal branch of the logarithm. Since $\lim _{z \rightarrow 0} z L_{0}\left(z^{2} /\left(z^{2}-1\right)\right)=0$, for $k$ large enough, the distance between $z\left[L_{0}\left(z^{2} /\left(z^{2}-1\right)\right)+2 \pi \mathrm{ik}\right]-1$ and $2 \pi i k z-1$ is less than $\frac{1}{2}$ for all $z$ on the circle $|2 \Pi i k z-1|=\frac{1}{2}$. Thus by Rouche's Theorem, for all sufficiently large $k$, the equation

$$
z\left[L_{0}\left(\frac{z^{2}}{z^{2}-1}\right)+2 \pi i k\right]=1
$$

has a solution $z_{k}$. Using the fact that $\lim _{z \rightarrow 0} z L_{0}\left(z^{2} /\left(z^{2}-1\right)\right)=0$ once again, we see that the sequences $\left\{\lambda_{k}\right\} \equiv\left\{z_{k}^{2} /\left(z_{k}^{2}-1\right)\right\}$ and $\left\{-1 / 4 \pi^{2} k^{2}\right\}$ are asymptotic. We now construct $\Gamma$ to spiral in from $\infty$ to 0 in such a way that it misses 1 and all the $\left\{\lambda_{k}\right\}$, and for $k$ sufficiently large, makes approximately one revolution about the origin between $\lambda_{k}$ and $\lambda_{k+1}$. By exercising some care, it is thus possible to have $L_{\Gamma}(1)=0$ and $z_{k} L_{\Gamma}\left(z_{k}^{2} /\left(z_{k}^{2}-1\right)\right)=1$ for infinitely many $k$. Finally, we note that the convergence of $\Sigma 1 / k^{2}$ allows us to make $\gamma=\zeta^{-1}(\Gamma)$ rectifiable. This completes the proof.

Remarks. Proposition 2 remains true if $1 / z$ is replaced by any rational function having a simple pole at 0 . On the other hand, it is impossible for $\int_{\gamma} d w /(w-z)$ to have infinitely many points of agreement with any rational function; a formal proof of this fact, which is somewhat intricate, is based on the intuitive idea that any $\Gamma$ for which the equation $z L_{\Gamma}(z /(z-1))=1$ has infinitely many solutions winds around the origin too often to allow $\gamma=\zeta^{-1}(\Gamma)$ to be rectifiable.

Finally, we remark that there is a closed $\gamma$ (with infinitely many self-intersections) for which the points of agreement of $\int_{\gamma} d w /\left(w-z^{2}\right)$ and $1 / z$ are precisely $\{1 / 2 \pi i k \mid$ $k=1,2, \ldots\}$. For the proof set $a_{k}=1 / 2 \pi i k, b_{k}=a_{k}^{2} /\left(a_{k}^{2}-1\right)$, and construct $\Gamma$ so that $L_{\Gamma}\left(b_{k}\right)=L_{0}\left(b_{k}\right)+2 \pi i k, k=1,2, \ldots$. The desired $\gamma$ is the union of $\zeta^{-1}(\Gamma)$ with the line segment joining 0 to 1 .

4. Remarks on the local nature of $\Phi_{\gamma}(r)$. In this section we will examine the nature of $r_{\gamma}$ and $\Phi_{\gamma}(r)$ in a neighborhood of a point $z_{0}$ not in $\Phi_{\gamma}(r)$. In such a neighborhood one would expect the complement of $\Phi_{\gamma}(r)$ to resemble a finite union of curves passing through $z_{0}$ and $r_{\gamma}$ to have an isolated logarithmic singularity at $z_{0}$. Below we will show that this is indeed the local picture for $r_{\gamma}$ and indicate how the local nature of $r_{\gamma}$ can be described in terms of the root functions of $q(z, w)=0$.

We assume $\gamma$ is a rectifiable arc and that $r$ has the form (0.1). We write

$$
q(z, w)=q_{0}(z) w^{m}+q_{1}(z) w^{m-1}+\cdots+q_{m}(z),
$$

where $q_{0}, \ldots, q_{m}$ are polynomials with $q_{0} \neq 0$.

One description of the complement of $\Phi_{\gamma}(r)=\Phi_{\gamma}\left(q^{-1}\right)$ is easily obtained. Let $S$ be the $m$-sheeted Riemann surface forming the domain of the algebraic function 
$w=w(z)$ defined by $q(z, w)=0$. Let $\pi$ denote the projection of $S$ onto $\hat{\mathbf{C}}_{z}$. Clearly

$$
\hat{\mathbf{C}}_{z} \backslash \Phi_{\gamma}(r)=\pi\left(w^{-1}(\gamma)\right)
$$

We will refine this description of $\hat{\mathbf{C}}_{z} \backslash \Phi_{\gamma}(r)$ locally in terms of the distinct root functions $w_{1}(z), \ldots, w_{m}(z)$ which constitute $w=w(z)$.

As in the proof of Theorem 1, we take $z_{0}=0$, and assume the root functions $w_{1}(z), \ldots, w_{m}(z)$ are meromorphic at $z=0$. (This involves some distortion since we needed to make the substitution $z=u^{\alpha}$.) In a deleted neighborhood $\Delta$ of the origin these root functions $w_{1}(z), \ldots, w_{n}(z)$ have convergent Laurent expansions. We arrange that $w_{1}, \ldots, w_{d}$ are all of the root functions satisfying $w_{j}(0) \in \gamma$. By shrinking $\Delta$ if necessary, it can be assumed that the values $w_{d+1}(z), \ldots, w_{m}(z)(z \in \Delta)$ do not lie on $\gamma$.

From equation (2.1),

$$
r_{\gamma}(z)=h_{0}(z)+\sum_{i=1}^{d} h_{i}(z) \int_{\gamma} \frac{d w}{w-w_{i}(z)}, \quad z \in \Delta \cap \Phi_{\gamma}(r),
$$

where $h_{0}, h_{1}, \ldots, h_{d}$ are meromorphic in $\Delta$ with the only possible pole at $z=0$.

The local nature of the integrals in (4.1) is described in the following proposition.

Proposition 3. Let $\gamma$ be a rectifiable arc in $\mathbf{C}_{w}$. Suppose $w=w(z)$ is a nonconstant analytic function defined in a neighborhood of the origin with $w(0)$ the initial point of $\gamma$. In a sufficiently small neighborhood $\Delta$ of $z=0$, the function

$$
W(z)=\int_{\gamma} \frac{d w}{w-w(z)}
$$

is analytic off a collection $\gamma_{1}, \ldots, \gamma_{k}$ of arcs whose only common point is their initial point $z=0$. Moreover,

$$
W(z)=\sum_{i=1}^{k} l_{\gamma_{i}}(z)+g(z), \quad z \notin \bigcup_{i=1}^{k} \gamma_{i},
$$

where $g$ is analytic in $\Delta$.

Proof. In a sufficiently small neighborhood $\Delta_{0}$ of $z=0$ we can write $w(z)=$ [ $\psi(z)]^{k}$, where $k \geqslant 1$ is an integer and $\lambda=\psi(z)$ is conformal mapping of $\Delta_{0}$ onto a neighborhood $V$ of $\lambda=0$ with $\psi(0)=0$.

Lift $\gamma$ to the $k$-sheeted Riemann surface for $w=\lambda^{k}$. Let $\gamma_{1}^{\prime}, \ldots, \gamma_{k}^{\prime}$ be the curves in the plane $C_{\lambda}$ which are the images of $\gamma$ under the $k$-branches of $\lambda=w^{1 / k}$. The curves $\gamma_{1}^{\prime}, \ldots, \gamma_{k}^{\prime}$ intersect only at $\lambda=0$. Moreover

$$
\int_{\gamma} \frac{d w}{w-\lambda^{k}}=\sum_{i=1}^{k} l_{\gamma_{i}^{\prime}}(\lambda), \quad \lambda \notin \bigcup_{i=1}^{k} \gamma_{i}^{\prime} .
$$

Choose arcs $\gamma_{i}^{\prime \prime}, \ldots, \gamma_{k}^{\prime \prime}$ of $\gamma_{1}^{\prime}, \ldots, \gamma_{k}^{\prime}$, respectively, such that $\gamma_{i}^{\prime \prime}$ is contained in $V$ and has initial point $\lambda=0$. Set $\gamma_{i}=\psi^{-1}\left(\gamma_{i}^{\prime \prime}\right)$. Then in a sufficiently small neighborhood $\Delta_{i}$ of $z=0$,

$$
l_{\gamma_{i}}(\psi(z))=l_{\gamma_{i}}(z)+g_{i}(z),
$$

where $g_{i}$ is analytic on $\Delta_{i}(i=1, \ldots, k)$. 
Let $\Delta=\bigcap_{i=1}^{k} \Delta_{i}$ and $g=g_{1}+\cdots+g_{k}$. The representation (4.2) follows by substituting (4.4) in (4.3). This ends the proof.

COROLlaRY 1. Let $\gamma$ be a rectifiable arc in $\mathbf{C}_{w}$ and $r(z, w)=p(z, w) / q(z, w) a$ quotient of relatively prime polynomials in $z, w$. Suppose $z=0$ is not in $\Phi_{\gamma}(r)$ and the root functions of $q(z, w)=0$ are all meromorphic at $z=0$. In a sufficiently small neighborhood $\Delta$ of the origin the function $r_{\gamma}$ is analytic off the union of a collection of simple curves $\gamma_{1}, \ldots, \gamma_{n}$ with initial point $z=0$. Moreover,

$$
r_{\gamma}(z)=f_{0}(z)+\sum_{i=1}^{n} f_{i}(z) l_{\gamma_{i}}(z), \quad z \in \Delta \cap \Phi_{\gamma}(r),
$$

where $f_{0}, \ldots, f_{n}$ are meromorphic at zero.

Proof. The result in the corollary follows easily when Proposition 3 is applied to each of the integrals in (4.1). In the cases where $w_{i}(0)$ is not an end of $\gamma$ it is necessary to write $\gamma=\gamma_{+}-\gamma_{-}$, where $\gamma_{ \pm}$are curves with initial point $w(0)$. This ends the proof.

We remark that when $\gamma$ is smooth the curves $\gamma_{1}, \ldots, \gamma_{n}$ appearing in the last corollary are smooth; however, if $\gamma$ is merely assumed to be rectifiable, then $\gamma_{1}, \ldots, \gamma_{n}$ may fail to be rectifiable. Further, the functions $f_{0}, \ldots, f_{n}$ and the curves $\gamma_{1}, \ldots, \gamma_{n}$ in this corollary may be explicitly given in terms of the root functions of $q(z, w)=0$. Finally, we note that by virtue of the representation of $r_{\gamma}$ given either as in Corollary 1 or as in the proof of Theorem 1 , it is clear that the only possible accumulation points of the isolated zeros of $r_{\gamma}$ are the solutions of $q(z, a)=0$ or $q(z, b)=0$, where $a, b$ are the ends of $\gamma$.

\section{REFERENCES}

1. E. Azoff, K. Clancey and I. Gohberg, On the spectra of finite-dimensional perturbations of matrix multiplication operators, Manuscripta Math. 30 (1980), 351-360.

2. Singular points of families of Fredholm integral operators Proceedings of the Toeplitz Memorial Conference, Operator Theory: Advances and Applications, Birkhauser-Verlag, Basel, (to appear).

Department of Mathematics, University of Georgia, Athens, Georgia 30602 (Current address of E. Azoff and K. Clancey)

Department of Mathematics, Tel-Aviv University, Ramat-Aviv, Tel-Aviv, IsRael (Current address of I. Gohberg) 